\title{
Celdas solares orgánicas, una perspectiva hacia el futuro
}

\author{
William Adolfo Chamorro Coral ${ }^{1 \star}$, Sara Urrego Riveros ${ }^{2 \star \star}$ \\ 1 Institut Jean Lamour, Département CP2S, UMR 7198 CNRS-Université de Lorraine, \\ Ecole des Mines de Nancy, Parc de Saurupt, CS14234, 54042 Nancy, France \\ 2 Universidad Nacional de Colombia, Departamento de Química, Cra. 30 No 45-03, \\ Bogotá, Colombia
}

Fecha de Entrega: Abril 13 DE 2012

Fecha de Evaluación: Abril 3 DE 2012

FECHA DE APROBACIÓN: MAYO 3 DE 2012

\begin{abstract}
Resumen La búsqueda de fuentes de energía alternativas a los combustibles fósiles ha permitido el desarrollo y uso de diferentes tecnologías basadas en el uso de la energía solar. Las celdas solares basadas en compuestos organicos ofrecen grandes ventajas en aspectos económicos, ambientales y tecnológicos y es por eso que la evolución que ha tenido desde sus comienzos ha permitido su paso hacia la producción a mediana escala por lo que sus perspectivas de crecimiento son muy alentadoras.
\end{abstract}

Palabras Clave: energía solar, evolución, celdas solares orgánicas, capa activa, p3HT, PCBM.

\begin{abstract}
The search for alternative energy sources to fossil fuels has enabled the development and use of different technologies based on the use of solar energy. Solar cells based on organic compounds offer great advantages in economic, environmental and technological, and that's why evolution has had since its begginings has enabled them to step to medium scale production so that its growth prospects are very encouraging.
\end{abstract}

Keywords: solar energy, evolution, organic solar cells, active layer, p3HT, PCBM.

\footnotetext{
* Químico con Maestría en ciencias (Química). Actualmente Candidato a Doctor en Ciencia e Ingeniería de Materiales. Áreas de interés: ZnO, pulverización catódica ( magnetron sputtering), defectos estructurales, difracción de rayos $\mathrm{X}$, fotoluminiscencia, plasmónica. william.chamorro@ijl.nancy-universite.fr

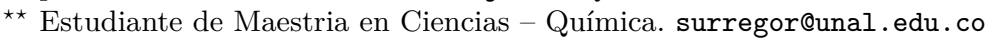




\section{Introducción}

Recientemente, en una cumbre mundial de energía se encontró que la demanda energética en el 2020 podría ser entre un $50 \%$ y $60 \%$ más alta que la actual, por lo cual el desarrollo de fuentes energéticas alternativas ha cobrado gran importancia. Entre estas fuentes alternativas se encuentran las celdas solares fotovoltaicas que permiten la generación de electricidad aprovechando la radiación del sol. Actualmente, la fabricación de módulos solares está basada en dos tecnologías principalmente, la primera es la denominada tecnología de Silicio mono- y policristalino o de primera generación, y la segunda es la denominada tecnología de películas delgadas o de segunda generación; esta última permite fabricar módulos FV a costos significativamente más bajos que los de Silicio, sin embargo estas dos tecnologías utilizan procedimientos costosos y complejos y utilizan como materia prima elementos poco abundantes o tóxicos, por lo cual se ha generado una gran expectativa en las tecnologías emergentes, que aunque aún no son competitivas económicamente frente a las tecnologías anteriores, tienen una gran proyección por las ventajas ofrecidas.

Es así como durante los últimos 40 años, la tecnología de las celdas solares inorgánicas basadas en Silicio y películas delgadas de sus derivados, se han posicionado como una alternativa energética al petróleo, puesto que utilizan una fuente energética gratuita y prácticamente inagotable, presentan altas eficiencias de conversión de energía radiante y su producción se realiza a escala industrial, sin embargo, sus altos costos de manufactura, los efectos negativos de algunos de sus componentes sobre el medio ambiente y la escases de la materia prima en la tierra, se presentan como serias limitantes para una mayor aplicación a nivel mundial. Las celdas solares basadas en polímeros semiconductores se presentan como una nueva alternativa, ya que las materias primas y los procesos de fabricación son de menor costo. Sin embargo es importante resaltar que a nivel local los materiales siguen teniendo costos elevados (por el transporte y los impuestos de importación), incrementando fuertemente el precio de la fabricación del dispositivo.

\section{2. ¿Es viable el uso de energía solar fotovoltaica?}

El descubrimiento del efecto fotovoltaico (PV) se atribuye comúnmente a Henry Becquerel, un físico francés, quien generó una fotocorriente al iluminar dos electrodos de platino, recubiertos con bromuro o cloruro de plata sumergidos en una solución acuosa. Smith y Adams hicieron los primeros informes de fotoconductividad, en 1873 y 1876, respectivamente, trabajando con selenio. Sin embargo solo hasta el principio de la década de 1950, los laboratorios Bell comenzaron a aplicar este efecto a través de celdas solares inorgánicas basadas en Silicio para producir energía eléctrica con una eficiencia del $6 \%$. Debido a la crisis energética que sufrió el mundo en la década de los 70, se generaron las oportunidades para que el uso de la energía solar entrara en el mercado de energías alternativas al petróleo [1]. Durante los últimos 30 años esta tecnología ha evolucionado por la base del 
amplio uso del Silicio en la microelectrónica, mejorando el diseño y fabricación. Recientemente se han estado desarrollando otras celdas basadas en películas delgadas de semiconductores con una o con múltiples junturas; esta evolución ha surgido con el fin de evitar el uso de grandes cantidades de materiales tóxicos: bajar el costo de la energía generada y aumentar la eficiencia de conversión hasta el 40,7\%. Con las mejoras realizadas y sus bajos costos, las celdas solares basadas en compuestos inorgánicos se han posicionado en el mercado de la energía aumentando su demanda en un $35 \%$ a $40 \%$ al llegar a producir $10.66 \mathrm{GW}$ en $2009[2,3]$.

Paralelamente a la evolución que han tenido las celdas basadas en compuestos inorgánicos, en la última década, han desarrollado celdas solares basadas en nuevos materiales fotovoltaicos entre las que se destacan las celdas sensibilizadas con colorantes o celdas DSSC también conocidas como electroquímicas, las celdas orgánicas basadas en polímeros conductores, las cuales han logrado eficiencias hasta de $8,3 \%$; su rendimiento teórico se ha encontrado que puede ser similar a los de los semiconductores tradicionales, lo que permite pensar que en el futuro su relación eficiencia/costo sea mayor que el de los otros tipos de celdas [2,3]. Por último se encuentran las celdas basadas en moléculas pequeñas las cuales se depositan por métodos físicos similares a las celdas solares inorgánicas.

Recientemente se ha estudiado la inclusión de láminas de nanotubos de carbono o grafeno como electrodo y/o capa buffer para mejorar el transporte eléctrico dentro de la celda solar orgánica [4].

En la figura 1 se muestra la evolución en el tiempo (durante los últimos 30 años) de la eficiencia de conversión de los diferentes tipos de celdas solares que se han desarrollado con tecnologías de primera, segunda y tercera generación. También aparecen los centros de investigación que mayor aporte han hecho para la consecución de estos resultados mostrando el aumento sostenido en la eficiencia de fotoconversión de luz solar y de la misma manera una contribución cada vez más grande de tecnologías y más atractivas tanto en el aspecto económico como ambiental $[2,3,5]$.

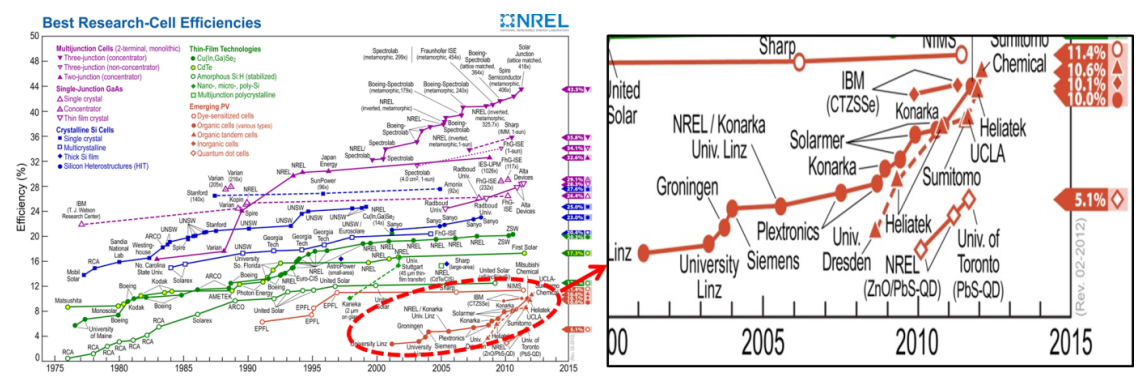

Figura 1. Evolución de las eficiencias para diferentes tecnologías en celdas solares. 


\section{Evolución de la tecnología de celdas solares orgánicas}

Una celda solar convencional consta de dos capas de material semiconductor, una positiva (tipo p) y una negativa (tipo $\mathrm{n}$ ), que se intercalan para formar una juntura $\mathrm{p} / \mathrm{n}$. Cuando el semiconductor está expuesto a la luz, la energía $(h \nu)$ de los fotones incidentes que excedan el umbral de energía (band gap) es absorbida por los electrones del semiconductor que tienen acceso a la banda de conducción empezando a conducir la electricidad. Para cada electrón, una carga positiva móvil o hueco es creada. Los electrones y huecos cerca de la juntura $\mathrm{p} / \mathrm{n}$ se dirigen en direcciones opuestas por la acción del campo eléctrico y al separarse las cargas, estos se dirigen hacia los electrodos respectivos, generando carga eléctrica [6]. Y aunque tradicionalmente se consideraban que los polímeros eran compuestos aislantes, a partir de los años 70 el químico japonés Hideki Shirakawa, el físico estadounidense Alan Heeger y el químico estadounidense, de origen neozelandés, Alan MacDiarmid realizaron trabajos en los cuales se descubrieron propiedades conductoras en cierto tipo de polímeros, hallazgo que les valió el premio nobel en química del año 2000 y que revolucionó la tecnología de los dispositivos electrónicos, desde las actuales pantallas AMOLED hasta los trabajos desarrollados en el campo de energía fotovoltaica, pero ¿qué es un polímero semiconductor?

Un polímero semiconductor es una macromolécula que presenta unidades repetitivas denominadas monómeros que, a diferencia de los polímeros tradicionales, son altamente conjugados, presentando dentro de su estructura electrones de orbitales $\pi$, lo que permite tener cadenas con una alta densidad electrónica deslocalizada, dándole propiedades electrónicas únicas, comparables a los semiconductores inorgánicos. A partir de este hallazgo se comienza a producir a manera de prototipo las primeras celdas solares y así empieza su evolución [7].

\subsection{Celdas solares orgánicas de una sola capa}

El primer modelo de CSO era relativamente simple, pues el primer tipo de celdas solares orgánicas consistía únicamente de una capa de un polímero conjugado entre dos electrodos metálicos que tenían como función la generación de un campo eléctrico. Debido a la baja constante dieléctrica de los polímeros conjugados, la primera fotoexcitación no libera electrones ni huecos, si no un excitón que es un par de enlaces entre electrón y hueco; al entrar en contacto con el cátodo por efecto Schottky, el excitón se disocia generando portadores de carga libre que se dirigen hacia sus respectivos electrodos, generando la corriente eléctrica. Estos excitones solo tienen unas decenas de $\mathrm{eV}$, lo que limita la eficiencia de conversión de potencia (PCE) a un $0,1 \%$. En la figura 2 se observa la composición y el diagrama de bandas de energía de este tipo de celdas [8].

\subsection{Celdas solares orgánicas bicapa (Concepto de heterojuntura)}

$\mathrm{Al}$ observar los bajos rendimientos en el anterior tipo de CSO se retomaron los conceptos y la misma configuración utilizada en las celdas solares inorgánicas, 


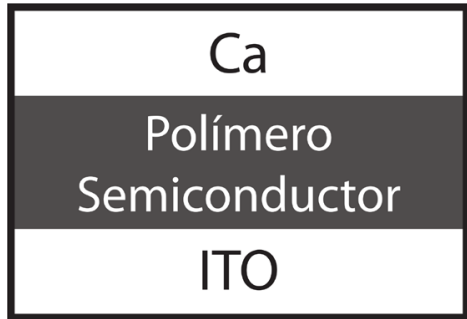

(a)

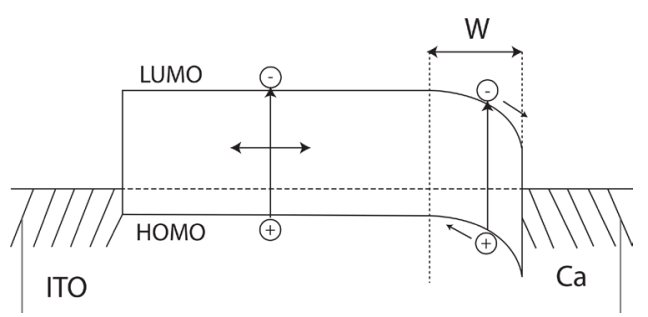

(b)

Figura 2. (a). Esquema de una celda solar de una sola capa. (b). Brecha de energía entre el HOMO y LUMO para una celda solar de una sola capa [8].

este nuevo tipo de celdas se basaba en la heterojuntura entre dos materiales con diferentes afinidades electrónicas y de potenciales de ionización (ver figura 3). Esto favorece la disociación del excitón en la interface de los dos materiales en electrones que serán aceptados por el material con mayor afinidad electrónica (material aceptor), y en huecos que se moverán a través del material con menor potencial de ionización (material donor), generando la corriente eléctrica tal como se mencionó en el proceso de funcionamiento. Sin embargo uno de los inconvenientes que presenta este tipo de celdas es que el exitón fotogenerado se disocia dentro de una longitud de difusión dentro de la interface donor/aceptor de aproximadamente $10 \mathrm{~nm}$, al fabricarse estas celdas, formando una capa de material sobre el otro, la distancia entre estos es mayor a $10 \mathrm{~nm}$, razón por la cual este tipo de celdas solo alcanzan un rendimiento de $3 \%$.

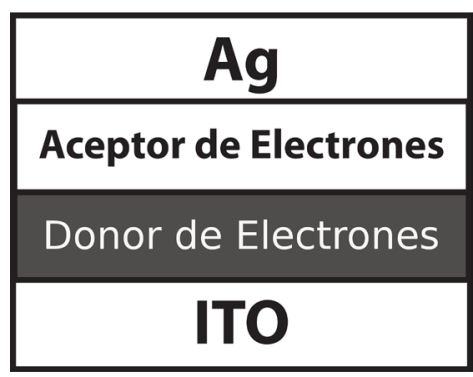

(a)

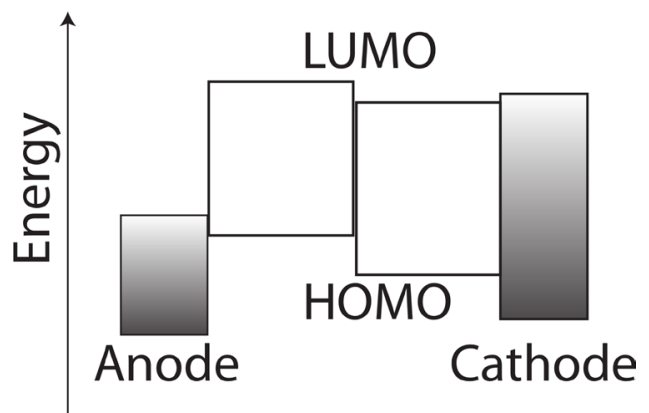

(b)

Figura 3. Celda solar con heterojuntura. (a). Esquema de una celda solar basado en la heterojuntura. (b). Disposición de las bandas de energía. 


\subsection{Heterojuntura en volumen o BHJ (Bulk Heterjunction)}

Con el mismo fin, de obtener mayores eficiencias, los diferentes estudios llevaron al desarrollo de un nuevo concepto de celda solar orgánica en la cual se corregían los inconvenientes de los anteriores tipos de celdas y toma fuerza el concepto de heterojuntura en volumen, este tipo de celdas tiene como capa activa una película delgada (100 nm aprox.) que está compuesta por una mezcla homogénea de un donor (polímero semiconductor altamente conjugado) y un aceptor de electrones (generalmente un derivado de fullereno), así en cualquier punto dentro de la película se tiene una mezcla donor/aceptor, resolviendo el inconveniente de las celdas con heterojuntura, puesto que las redes interpenetrantes aumentan las interfaces entre los dos materiales, disminuyendo la distancia entre ellos, favoreciendo el proceso de disociación del excitón y disminuyendo los procesos de recombinación, lo que incrementa la eficiencia de las celdas solares orgánicas. Sin embargo es preciso describir de manera más detallada los componentes de esta capa activa.

La máxima eficiencia de conversión reportada para una celda solar de este tipo, de 8,3\%, usa como capa activa la mezcla de P3HT [poly(3-hexylthiophene)] y el derivado del fullereno PCBM [Phenyl-C61-Butyric-Acid-Methyl-Ester], materiales donor y aceptor de electrones respectivamente. En la figura 4 se observa la sección trasversal de celdas tipo BHJ. En este tipo de celdas la luz atraviesa el sustrato transparente (vidrio alcalino o polietileno), y es absorbida en su mayoría por parte de la capa activa, dando lugar a la generación de la fotocorriente dentro del dispositivo. Por su parte, el contacto inferior debe tener propiedades adecuadas para conducir los huecos generados, generalmente se usa PEDOT:PSS sobre ITO, siendo el primero el que facilita el trasporte de huecos desde la capa activa hasta el segundo, pues es un semiconductor inorgánico transparente que actúa como ánodo. Finalmente una película de aluminio actúa como contacto superior.

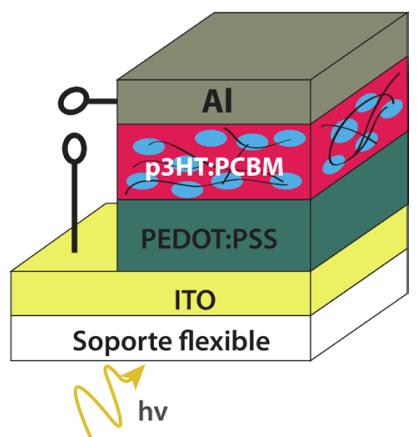

Figura 4. Sección transversal de una celda solar con estructura heterojuntura en el volumen. 


\subsection{Capa activa}

Donor de electrones. Teniendo en cuenta la facilidad con que se puede aumentar la conjugación de electrones $\pi$, se observa que un aumento en la conjugación permite que los niveles energéticos (orbitales) de los polímeros estén cada vez más cerca entre sí, de manera que se forme una estructura de "bandas" similar a la observada en lo semiconductores inorgánicos de estado sólido, que genera un ancho de banda prohibida (Eg) entre la "banda de valencia" llamada HOMO (High Occupied Molecular Orbital) y la "banda de conducción" LUMO (Low Unoccupied Molecular Orbital), lo que permite que se comporte como un semiconductor clásico $[1,7]$. Algunos polímeros semiconductores que tienen aplicación en dispositivos fotovoltaicos como material donor, se muestran en la figura 5.

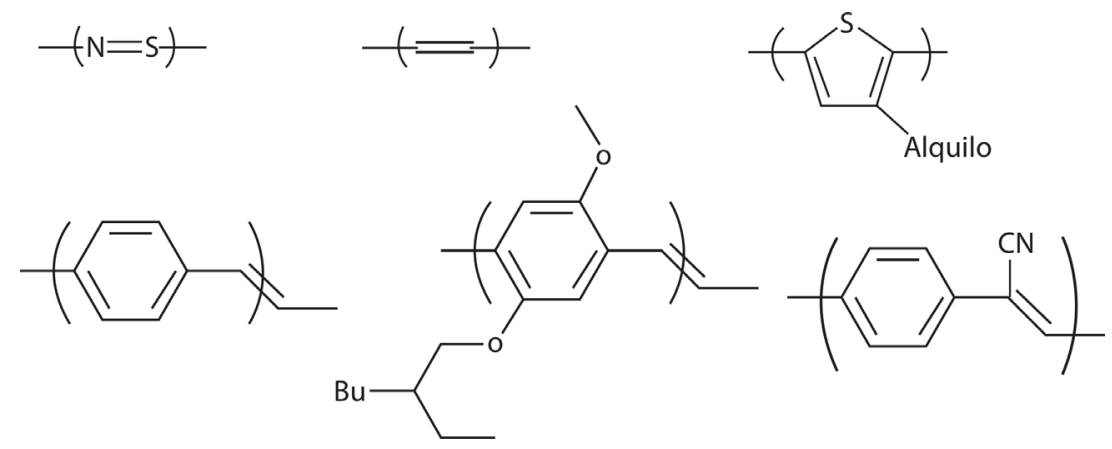

Figura 5. Algunos polímeros conjugados investigados en celdas PV. Superior: poli(nitruro de azufre) $\left(\mathrm{SN}_{\mathrm{x}}\right)$, poli-acetileno y el poli-(3-alquil-tiofeno). Inferior: poli-(pfenilenovinileno) (PPV), poli-(2-metoxi-5-(2'-etilhexiloxy)-1,4-fenilvinileno) (MEH$\mathrm{PPV})$, ciano-PPV (CN-PPV) [9]

Los polímeros semiconductores con propiedades fotovoltaicas de tipo donor de electrones, deben presentar las siguientes propiedades $[7,10,11,12,13,14]$ :

- Alta conductividad (mayor a 1,000 $\mathrm{S} \mathrm{cm}^{-1}$ )

- Altas movilidades de los huecos generados $\left(0.05-0.1 \mathrm{~cm}^{2} \mathrm{~V}^{-1} \mathrm{~s}^{-1}\right)$

- No presentar procesos radiativos

- Una diferencia energética entre HOMO y LUMO, banda prohibida $(E g)$ óptima que permita la mayor absorción de radiación posible. $(<2 \mathrm{eV})$

- Un coeficiente de absorción alto $\left(>105 \mathrm{~cm}^{-1}\right)$

- Alta solubilidad en solventes volátiles para facilitar la formación de la película, por las diferentes técnicas, como por ejemplo spin coating o cualquiera de tipo impresión roll to roll, doctor Blade, etc.

Estas propiedades se pueden mejorar mediante el uso de diferentes materiales, que a través de su síntesis, logren generar alta conjugación dentro de su estructura 
o que al ser solubles en solventes adecuados y entremezclarse con el material aceptor, se logre obtener una morfología adecuada para que existan los procesos de generación de corriente y así sean de gran utilidad en las celdas solares orgánicas.

El polímero ampliamente utilizado para su uso en celdas solares orgánicas es el P3HT que gracias a su estructura cumple con las anteriores propiedades, sin embargo las investigaciones se encaminan a obtener materiales donde se aumente la solubilidad, y se mejore la absorción de luz, para que se facilite así, el uso de técnicas de fabricación en solución (spin coating, roll to roll etc.). Lo anterior resulta en prácticas para producción a gran escala y de bajo costo. [10]

Aceptor de electrones. En la capa activa de la mezcla se tiene tanto el material donor de electrones (P3HT) como de un material que acepte estos electrones, con el fin de dar paso a la generación de corriente. Como vimos anteriormente, el P3HT cumple la función de absorber la mayor cantidad posible de radiación proveniente del sol, en esta etapa los electrones son excitados; sin embargo la conversión de luz solar en corriente eléctrica necesita de un paso adicional, la trasferencia de estos electrones, para lo cual es necesario hacer uso de un material aceptor de electrones que evite las pérdidas en procesos radiativos, es decir que evite la recombinación del excitón.

Actualmente el PCBM, al ser un derivado del fullereno, tiene una alta conductividad eléctrica y sus niveles de energía HOMO y LUMO se ajustan a los del P3HT; además este derivado es más soluble que el fullereno porque permite tener una mayor homogeneidad en la mezcla lo cual también beneficia las propiedades de trasporte eléctrico, ya que se encuentra entremezclado correctamente con el material aceptor [15].

En la figura 6 podemos observar la estructura química de cada uno de los dos compuestos.
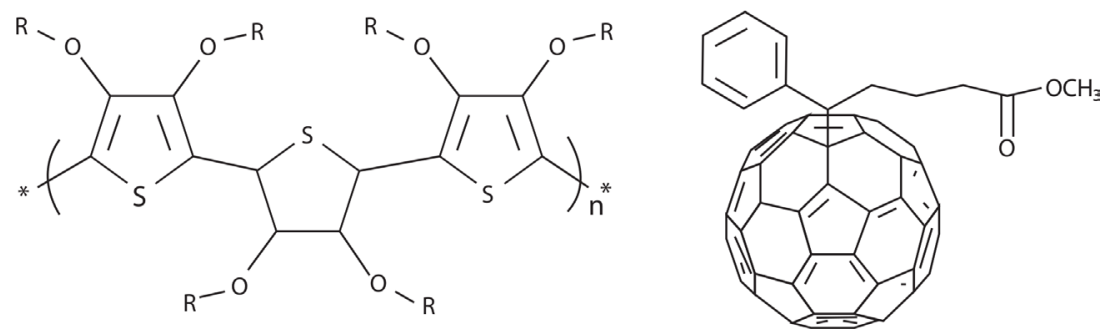

Figura 6. Estructuras químicas del P3HT (izquierda) y del PCBM (derecha). 


\section{Y ¿cómo funcionan?}

Para entender el principio de operación en la figura 7, se muestra el diagrama de bandas de energías del material donor y aceptor, donde se observa que en las CSO, la absorción de fotones (a) por parte de la capa activa, genera una transición electrónica entre estados HOMO y LUMO del material donor, generando un sistema de pares electrón-hueco conocidos como excitones (b), localizados en la estructura del compuesto; los exitones generados se difunden hasta la interface con el material aceptor donde se disocian generando portadores de carga libres (c) que se mueven independientemente a través de cada material hasta llegar a los contactos eléctricos (d). Para alcanzar la separación de la carga es necesario un campo eléctrico, que es proporcionado por la asimetría de la energía de ionización/funciones-trabajo de los electrodos. Esta asimetría es la razón del porqué el flujo de electrones se ve favorecida desde el electrodo con menor función trabajo hacia el electrodo con mayor función-trabajo (polarización directa) $[1,8]$. Este tipo de celdas solares presentan como inconveniente procesos de recombinación del excitón, ya sea porque no alcance a llegar a la interface, generando dentro del material donor procesos de luminiscencia por recombinación geminal, o puede ser que se recombinen los huecos y electrones disociados en la interface, ya que al estar muy cerca estos no alcancen a difundirse en sus respectivos materiales y genere también un efecto luminiscente dentro de toda la mezcla.

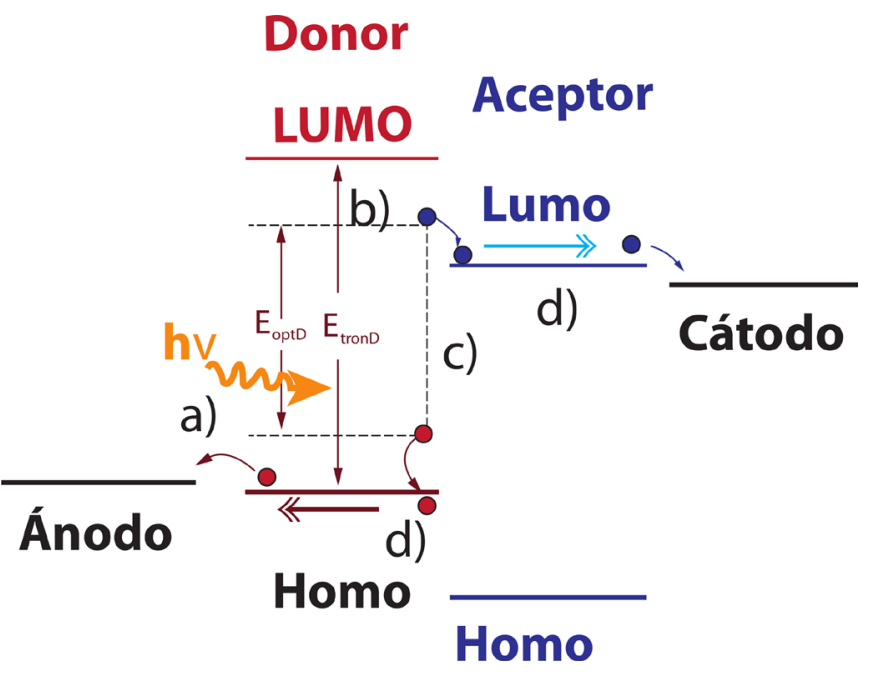

Figura 7. Procesos que ocurren dentro de la capa absorbente de una celda solar orgánica. 
De una manera más detallada, en la figura 8 se puede observar cómo es el movimiento de los electrones desde el material donor hacia el aceptor.

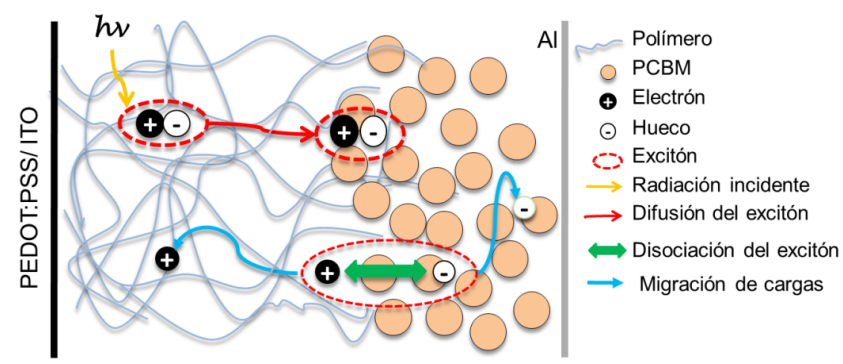

Figura 8. Esquema de la generación de carga en las celdas solares orgánicas BHJ.

\section{Retos a superar}

Los resultados obtenidos con las celdas solares orgánicas son positivos, ya que actualmente existen empresas produciendo módulos de celdas orgánicas a baja escala, sin embargo aún se está en la búsqueda de la optimización de las mezclas de capas activas.

A partir de la síntesis de polímeros más eficientes con reactivos de menor costo y fácil acceso, es necesario resolver varios de los problemas inherentes con la heterojuntura en solución (con el PCBM) como la miscibilidad en estado sólido. Los sistemas conjugados grandes y extendidos no son normalmente miscible por lo que se buscan compuestos que sean solubles; así mismo la fabricación de este tipo de celdas se basa en técnicas en las cuales el solvente se debe evaporar rápidamente, como en la técnica de spin coating, y de esta manera obtener películas delgadas y homogéneas de capa activa par poder disminuir los procesos radiativos (fluorescencia) y ampliar el rango de absorción del espectro solar. Otro de los proyectos a corto plazo es disminuir las pérdidas de corriente debidos a los contactos eléctricos y se ha propuesto utilizar capas del popular grafeno, que mejoraría la eficiencia de las CSO por ser un buen conductor, pero además por facilitar la movilidad de los huecos generados en la capa activa.

Las perspectivas muestran que las investigaciones que se realizan actualmente a nivel mundial, generarán una disminución de costos y un menor impacto al medio ambiente permitiendo así, un desarrollo cada vez más viable puesto que se generarían dispositivos que cumplen con los requisitos de la búsqueda de energías alternativas, eficientes, limpias y económicas, a partir de un recurso inagotable, la energía solar, por lo que la permitirán posicionar en un futuro próximo este tipo de tecnologías en cada hogar. 


\section{Referencias}

1. Spanggaard, H., Krebs, F.: Solar Energy Materials \& solar cells 83 125-146 (2004)

2. Razykov, T.M. et al.: Solar Energy, Volume 85 1580-1608 (2011)

3. Parida, B.. et al.: Renewable and sustainable energy reviews 15 1625-1636 (2011)

4. Kim, Y.H., et al.: Solar Energy materials \& solar cells, 96 244-250 (2012)

5. http://www.nrel.gov/ncpv/images/efficiency_chart.jpg citado el 30 de marzo de 2011 (2011)

6. Pagliaro, M.: Flexible Solar Cells, Wiley VCH, 31-32, 85-86 p. (2008)

7. Nobel price organization, Heeger, A., MacDiarmid, A., Shirakawa, H.: The Nobel Prize in Chemistry, 2000: Conductive polymers - advanced information, [en línea] http://www.nobelprize.org/nobel_prizes/chemistry/laureates/2000/ chemadv.pdf, citado el 1 de julio de 2011 (2011)

8. Hoppe, H., Sariciftci, N.: J.Mat.Res. vol.19, No.7, 1924-1945 (2004)

9. Chen, J., Cao, Y.: Accounts of chemical research, Vol 42, No.11 1709-1718. (2009)

10. Krebs, F.: Solar Energy Materials \& Solar Cells 93 394-412 (2009)

11. Deibel, C., Dyakonov.: Rep.Prog.Phys.73 096401 (39pp) (2010)

12. Cheng, Y., et al: Chem. Rev. 109, 5868-5923 (2009)

13. Tracey, M., et al.: Chem. Rev. 110, 6736-6767 (2010)

14. Wang, et al.: Solar Energy materials \& solar cells 98 129-145 (2012)

15. Mikroyannidis, J.A., Kabanakis, A.N., Sharma, S.S., Sharma, G.D.: Adv. Funct. Mater. 21: 746-755. (2011) 